B.S. KOMAL AND P.S. SINGH

KODAI MATH. J.

14 (1991), 463-469

\title{
COMPOSITION OPERATORS ON THE SPACE OF ENTIRE FUNCTIONS
}

\author{
By B. S. Komal and Prem SAgar Singh
}

\begin{abstract}
The composition operators on the space of entire functions $\Gamma$ have been characterized. The invertibility of a composition operator $C_{\phi}$ interms of the invertibility of inducing map $\phi$ is obtained.
\end{abstract}

\section{Preliminaries.}

Let $X$ be a non-empty set and let $V(X)$ be a vector space of complex valued functions on $X$. If $\phi: X \rightarrow X$ is a mapping such that $f^{\circ} \phi \in V(X)$ whenever $f \in V(X)$, then a composition transformation $C_{\phi}$ is defined by the equation

$$
C_{\phi} f=f^{\circ} \phi \quad \text { for every } f \in V(X) .
$$

In case $V(X)$ is a topological vector space and $C_{\phi}$ is continuous, then we call it a composition operator induced by $\phi$. If $u: X \rightarrow C \backslash\{0\}$ is a mapping such that $\left(u C_{\phi}\right) f=u . f \circ \phi \in V(X)$ whenever $f \in V(X)$, then a weighted composition operator is a continuous linear transformation $u C_{\phi}: V(X) \rightarrow V(X)$ defined by

$$
\left(u C_{\phi}\right) f=u . f \circ \phi \quad \text { for every } f \in V(X) .
$$

A complex valued function $f: C \rightarrow C$ of a complex variable is called an entire function if it is analytic in the whole complex plane. If $f$ is an entire function then there exists a sequence $\left\{\hat{f}_{n}\right\}$ of complex numbers such that

$$
\left|\hat{f}_{n}\right|^{1 / n} \longrightarrow 0 \quad \text { as } n \rightarrow \infty \text { and } f=f(z)=\sum_{n=0}^{\infty} \hat{f}_{n} z^{n}
$$

The power series in (1) is a uniformly convergent power series. Conversely every sequence $\left\{\hat{f}_{n}\right\}$ of complex numbers with $\left|\hat{f}_{n}\right|^{1 / n} \rightarrow 0$ as $n \rightarrow \infty$ defines an

(1980) AMS (MOS) - Subject Classification (1985: Revision), Primary: 47B38, Secondary 30D10.

Keywords and Phrases. Entire function, Uniform convergence on compact sets, Composition operator, Invertible and isometric operators.

This work is partially supported by U.G.C. Grant NO: F-8-5(30)/87 SR II.

Received October 19, 1990 ; revised April 11, 1991. 
entire function $f$ represented by (1). We can define a metric $d$ in the class of entire functions as $d(f, g)=\sup \left\{\left|\hat{f}_{0}-\hat{g}_{0}\right|,\left|\hat{f}_{n}-\hat{g}_{n}\right|^{1 / n}, n \geqq 1\right\}$. The class of entire functions topologized by this metric is denoted by $\Gamma$. It is shown in Iyer [8] that $\Gamma$ is a non-normable complete metrizable locally convex topological vector space. The convergence of a sequence of entire functions in the metric topology of $\Gamma$ is equivalent to the uniform convergence of entire functions in any circle of finite radius. Such a convergence in $\Gamma$ will be called strong convergence in $\Gamma$.

Every continuous linear functional $f$ on $\Gamma$ is given by $f(\alpha)=\sum_{n=0}^{\infty} f_{n} a_{n}$, where $\alpha=\alpha(z)=\sum_{n=0}^{\infty} a_{n} z^{n}$ and $\left\{f_{n}\right\}$ is a sequence of complex numbers such that $\left\{\left|f_{n}\right|^{1 / n}\right\}$ is a bounded sequence. The set of all bounded linear functionals on $\Gamma$ is denoted by $\Gamma^{*}$. A sequence $\left\{\alpha_{n}\right\}$ in $\Gamma$ is said to converge weakly to $\alpha \in \Gamma$ if and only if $f\left(\alpha_{n}\right) \rightarrow f(\alpha)$ for every $f \in \Gamma^{*}$. If for each $n \in Z_{+}$, we define $e_{n}: C \rightarrow C$ as $e_{n}(z)=z^{n}$, then the sequence $\left\{e_{n}: n \in Z_{+}\right\}$is a basis for $\Gamma$. A sequence $\left\{\alpha_{n}\right\}$ in $\Gamma$ is called basis for $\Gamma$ if for each $\alpha \in \Gamma$ there exists a unique sequence $\left\{t_{n}(\alpha)\right\}$ of complex number such that $\alpha=\sum_{n=0}^{\infty} t_{n}(\alpha) \alpha_{n}$. The space $\Gamma$ of entire functions has been studied extensively by Iyer ([9], [10] and [11]).

In this note we plan to study composition operators on $\Gamma$. Most of the work on composition operators is done on Hardy spaces and $L^{p}$-spaces. Nordgren [13] has summarized some known information about composition operators on $L^{2}$ and $H^{2}$ spaces. For further details about these operators we refer to Schwartz [8], Swantan [9], Cowen [6], Boyd [2], Iwanik Mayer [12], Singh [16] and Singh and Komal [17]. The weighted composition operators have been studied by Carlson [3].

We have characterized composition operators on $\Gamma$. The invertibility of $C_{\phi}$ in terms of the invertibility of $\phi$ is reported. Weighted composition operators on $\Gamma$ have also been characterized. For $R>0$, we denote by $D_{R}$ the open disc $\{z \in C:|z|<R\}$. If $f \in \Gamma$, then $M(R, f)=\sup \left\{|f(z)|: z \in \bar{D}_{R}\right\}$. For $z \in C$, the evaluation functional is a map $E_{z}: \Gamma \rightarrow C$ defined by $E_{z}(f)=f(z)$ for every $f \in \Gamma$. The symbol $C(T)$ denotes the set of continuous linear operators on $\Gamma$ into itself.

\section{Characterizations of composition operators.}

In this section we obtain some characterizations of composition operators. We first prove the following lemma:

LEMmA 2.1. Let $R>0$. Then for each $z \in D_{R}$ and $f \in \Gamma$,

$$
|f(z)| \leqq \frac{R M(R, f)}{R-|z|} .
$$


Proof. By Cauchy integral formula

$$
f(z)=\frac{1}{2 \pi i} \int_{C_{R}} \frac{f(w) d w}{w-z},
$$

where $C_{R}$ is the circle $|w|=R$. Hence

$$
\begin{aligned}
|f(z)| & \leqq \frac{1}{2 \pi} \int_{C_{R}} \frac{|f(w)||d w|}{|w|-|z|} \\
& \leqq \frac{M(R, f)}{2 \pi} \int_{C_{R}} \frac{|d w|}{R-|z|} \\
& =\frac{R M(R, f)}{R-|z|}
\end{aligned}
$$

THEOREM 2.2. Let $\phi: C \rightarrow C$ be a mapping. Then $C_{\phi} \in C(\Gamma)$ if and only if $\phi$ is an entire function.

Proof. Suppose $\phi$ is an entire function. Since composition of two entire functions is an entire function, so $f \circ \phi$ is an entire function for each $f \in \Gamma$. We prove that $C_{\phi}$ is continuous. It is enough to prove that $C_{\phi}$ is continuous at origin. Let $R>0$ be given. Then $\bar{D}_{R}$ is a compact subset of $C$. But $\phi$ is continuous. Therefore $\phi\left(\bar{D}_{R}\right)$ is also compact subset of $C$. Hence we can find $K \geqq M(R, \phi)$ such that $\phi\left(\bar{D}_{R}\right) \subset \bar{D}_{K}$. Now convergence in $\Gamma$ is equivalent to uniform convergence in any circle of finite radius. Suppose $f_{n \rightarrow 0}$ strongly. Then for each $\varepsilon>0$ we can find some $n_{0}>0$ such that $M\left(K, f_{n}\right)<\varepsilon K_{0} / K$ where $K_{0}=K-M(R, \phi)$, for all $n \geqq n_{0}$. From Lemma 2.1, we have

$$
\begin{aligned}
\left|f_{n}(\phi(z))\right| & \leqq \frac{K M\left(K, f_{n}\right)}{K-|\phi(z)|} \\
& \leqq \frac{K M\left(K, f_{n}\right)}{K-M(R, \phi)}<\varepsilon, \quad \text { for every } z \in D_{R} \text { and for all } n \geqq n_{0} .
\end{aligned}
$$

Hence $C_{\phi} f_{n}=f_{n} \circ \phi \rightarrow 0$ as $n \rightarrow \infty$.

Conversely, suppose $C_{\phi}: \Gamma \rightarrow \Gamma$ is continuous. Then $C_{\phi} f=f \circ \phi$ is an entire function for every $f \in \Gamma$. In particular, take $f=I$. Then $\phi=I \circ \phi=f \circ \phi$. Hence $\phi$ is an entire function.

TheOREM 2.3. Let $A \in C(\Gamma)$. Then $A$ is a composition operator if and only if $A e_{n}=\left(A e_{1}\right)^{n}$ for every $n \in Z_{+}$.

Proof. Suppose $A$ is a composition operator. Then $A=C_{\phi}$ for some entire function $\phi: C \rightarrow C$. Therefore

$$
\begin{aligned}
A e_{n} & =C_{\phi} e_{n}=e_{n} \circ \phi=\phi^{n}=\left(e_{1} \circ \phi\right)^{n} \\
& =\left(C_{\phi} e_{1}\right)^{n}=\left(A e_{1}\right)^{n} \quad \text { for every } n \in Z_{+} .
\end{aligned}
$$


Conversely, if the condition of the theorem is satisfied, then set $\phi=A e_{1}$. Clearly $\phi$ is an entire function. Hence $C_{\phi}$ is a composition operator. Now

$$
\begin{aligned}
& A f=A\left(\sum_{n=0}^{\infty} \hat{f}_{n} e_{n}\right)=\sum_{n=0}^{\infty} \hat{f}_{n} A e_{n} \\
& =\sum_{n=0}^{\infty} \hat{f}_{n}\left(A e_{1}\right)^{n}=\sum_{n=0}^{\infty} \hat{f}_{n} \phi^{n} \\
& =\sum_{n=0}^{\infty} \hat{f}_{n} e_{n} \circ \phi=\sum_{n=0}^{\infty} \hat{f}_{n} C_{\phi} e_{n} \\
& =C_{\phi}\left(\sum_{n=0}^{\infty} \hat{f}_{n} e_{n}\right)=C_{\phi} f, \quad \text { for every } f \in \Gamma . \text { Therefore, } \\
& \qquad A=C_{\phi} .
\end{aligned}
$$

Theorem 2.4. Let $A \in C(\Gamma)$. Then $A$ is a composition operator if and only if $A^{*} E \subset E$, where $E=\left\{E_{z}: z \in C\right\}$.

Proof. For each $z \in C$, the evaluation functional $E_{z} \in \Gamma^{*}$ in view of Lemma 2.1. Since

$$
\left(C_{\psi}^{*} E_{z}\right) f=E_{z}\left(C_{\phi} f\right)=(f \circ \phi)(z)=f(\phi(z))=E_{\phi(z)}(f)
$$

for every $f \in \Gamma$, so $C_{\phi}^{*}(E) \subset E$. Hence if $A=C_{\phi}$, then $A^{*}(E) \subset E$.

Conversely, if $A^{*} E_{z}=E_{w}$ for some $w \in C$, then define $\phi(z)=w$. Now

$$
\begin{aligned}
(A f)(z) & =E_{z}(A f)=A^{*}\left(E_{z}\right) f \\
& =E_{w}(f)=E_{\phi(z)} f \\
& =f(\phi(z))=\left(C_{\phi} f\right)(z)
\end{aligned}
$$

for every $z \in C$ and $f \in \Gamma$. Hence $A=C_{\phi}$.

Theorem 2.5. Let $C_{\phi} \in C(\Gamma)$. Then $C_{\phi}^{*}: \Gamma^{*} \rightarrow \Gamma^{*}$ is a composition operator if $\phi(z)=\alpha z$.

Proof. Suppose $\phi(z)=\alpha z$. Define $\phi: C \rightarrow C$ by $\phi(z)=\alpha z$. We prove that $C_{\phi}^{*}=C_{\psi}$. Let $f \in \Gamma^{*}$ and $x \in \Gamma$. Then $f(z)=\sum_{n=0}^{\infty} f_{n} z^{n}$ and $x(z)=\sum_{n=0}^{\infty} \hat{x}_{n} z^{n}$. Therefore, $\quad x(\phi(z))=\sum_{n=0}^{\infty}\left(\hat{x}_{\circ} \phi\right)(n) z^{n}$. But $x(\phi(z))=\sum_{n=0}^{\infty} \hat{x}_{n}(\phi(z))^{n}=\sum_{n=0}^{\infty} \hat{x}_{n}(\alpha z)^{n}=\sum_{n=0}^{\infty} \alpha^{n} \hat{x}_{n} z^{n}$. Hence by unique expansion of $x(\phi(z))$, we have $(\hat{x} \circ \phi)(n)=\alpha^{n} \hat{x}_{n}$. Similarly $f(\phi(z))=$ $f(\alpha z)=\sum_{n=0}^{\infty} \alpha^{n} f_{n} z^{n}$. Now $\left(C_{\phi}^{*} f\right)(x)=f\left(C_{\phi} x\right)=\sum_{n=0}^{\infty} f_{n}(\hat{x} \circ \phi)(n)=\sum_{n=0}^{\infty} \alpha^{n} f_{n} \hat{x}(n)=(f \circ \phi)(x)$ $=\left(C_{\psi} f\right)(x)$ for every $x \in \Gamma$ and $f \in \Gamma^{*}$. Therefore $C_{\phi}^{*}=C_{\psi}$. 


\section{Invertible composition operators.}

A continuous linear transformation $A: \Gamma \rightarrow \Gamma$ is called invertible if there exists a continuous linear transformation $B: \Gamma \rightarrow \Gamma$ such that $A \circ B=B \circ A=I$, the identity operator on $\Gamma$. Similarly a mapping $\phi: C \rightarrow C$ is called invertible if there exists a mapping $\phi: C \rightarrow C$ such that $\phi \circ \psi=\psi \circ \phi=I$, the identity mapping on $C$. Let $A \in C(\Gamma)$. Then $A$ is called an isometry if $d(A f, A g)=d(f, g)$ for every $f, g \in \Gamma$. In this section invertible and isometric composition operators have been studied.

Theorem 3.1. Let $C_{\phi} \in \Gamma$. Then $C_{\phi}$ is invertible if and only if $\phi$ is invertible with $\phi^{-1} \in \Gamma$.

Proof. Suppose $C_{\phi}$ is invertible. Then thre exists $A \in C(\Gamma)$ such that $A C_{\phi}=C_{\phi} A=I$. So we have

$$
\begin{aligned}
A e_{n} & =A e_{1}^{n}=A\left(\left(C_{\phi} A e_{1}\right)^{n}\right)=A\left(\left(\left(A e_{1}\right) \circ \phi\right)^{n}\right) \\
& =A\left(\left(A e_{1}\right)^{n} \circ \phi\right)=A C_{\phi}\left(\left(A e_{1}\right)^{n}\right) \\
& =\left(A e_{1}\right)^{n} \quad \text { for } n=0,1,2, \cdots .
\end{aligned}
$$

By theorem 2.2A $A=C_{\psi}$ for some entire function $\phi$. It follows that $\phi \circ \phi=\phi \circ \phi=I$. This atonce implies that $\phi$ and $\phi$ are bijections. Hence $\phi^{-1} \in \Gamma$.

To prove the converse, let $\phi$ be invertible with $\phi^{-1} \in \Gamma$. Then $C_{\phi^{-1}}$ is a composition operator. Clearly $C_{\phi} C_{\phi^{-1}}=C_{\phi^{-1}} C_{\phi}=I$. Hence $C_{\phi}$ is invertible.

CoRollary 3.2. Let $C_{\phi} \in C(\Gamma)$. Then $C_{\phi}$ is invertible if and only if $\phi(z)$ $=a z+b$, where $(0 \neq) a, b \in C$.

Proof. By Theorem $3.1 C_{\phi}$ is invertible if and only if $\phi$ is bijective on $C$. And this is the case if and only if $\phi(z)=a z+b$ with $a \neq 0$. (In fact, if $\phi$ is a polynomial, then it should be linear. If it is not a polynomial, it has an essential singularity at the point at infinity, so that it can not be one-to-one).

THEOREM 3.3. Let $C_{\phi} \in C(\Gamma)$. Then $C_{\phi}$ is an isometry if and only if $\phi(z)$ $=\alpha z$ where $|\alpha|=1$.

Proof. Let $C_{\phi}$ be an isometry. Then, $d\left(C_{\phi}\left(e_{1}\right), 0\right)=d\left(e_{1}, 0\right)=1$, so that we have $|\hat{\phi}(0)| \leqq 1$ and $|\hat{\phi}(n)|^{1 / n} \leqq 1$ for $n=1,2, \cdots$. Also, $d\left(C_{\phi}(z+c), 0\right)=d(z+c, 0)$ $=\max \{1,|c|\}$. If $|c|>2$, then $|c+\hat{\phi}(0)| \leqq|c|$. This means that $\hat{\phi}(0)=0$. Next, suppose $\hat{\phi}(m) \neq 0$ for some $m \geqq 2$. Since $d\left(C_{\phi}\left(\alpha e_{1}\right), 0\right)=d\left(\alpha e_{1}, 0\right)=|a|$ implies that $|\alpha \hat{\phi}(m)|^{1 / m} \leqq|\alpha|$ or $|\alpha| \leqq|\alpha|^{m} /|\hat{\phi}(m)|$, which yields a contradiction by letting $\alpha \rightarrow 0$. Hence, $\phi(z)=\hat{\phi}(1) z$. That $|\hat{\phi}(1)|=1$ follows at once from the identity $d(\phi, 0)=d\left(C_{\phi}\left(e_{1}\right), 0\right)=d\left(e_{1}, 0\right)=1$.

Conversely if $\phi(z)=\alpha z$ for some $\alpha \in C$ such that $|\alpha|=1$, then clearly $C_{\phi}$ is 
an isometry.

\section{Weighted composition operators on $\Gamma$.}

A caracterization of weighted composition operators is obtained in this section.

THEorem 4.1. Let $u: C \rightarrow C$ and $\phi: C \rightarrow C$ be two non-trivial mappings. Then $u C_{\phi} \in C(\Gamma)$ if and only if $u$ and $\phi$ are entire functions.

Proof. First we suppose that $u C_{\phi}$ is a continuous linear operator. Then $u . f \circ \phi$ is an entire function for every entire function $f$. Now, if we take $f$ to be a constant function which is equal to 1 every where, then we have $u . f \circ \phi$ $=u$ so that $u$ is an entire function. Further, if we take $f=I$, the identity function then $u . f \circ \phi=u$. Suppose $u \neq \equiv$. By the assumption we see that $u(z)(\phi(z))^{n}=u C_{\phi}\left(e_{n}\right)$ is entire for every $n=0,1,2, \cdots$. That $u$ is entire follows from the case $n=0$. The case $n=1$ shows that $\phi(z)$ is analytic wherever $u(z)$ $\neq 0$. Suppose that $u$ has a zero of order $m>0$ at a point $\alpha$. If $\phi(z)$ has a pole of order $k$ at the point $\alpha$, then $C_{\phi}\left(e_{n}\right)$ has a pole of order $n k$ there. So, for $n$ with $n k>m$ the function $u C_{\phi}\left(e_{n}\right)$ cannot be analytic at $\alpha$. In case $\alpha$ is an essential singularity for $\phi, u \phi$ cannot be analytic at $\alpha$. This means that $\phi$ should be analytic at $\alpha$. Hence, $\phi$ is an entire function.

To prove the converse, let $u$ and $\phi$ be entire functions. Since product and composition of two entire functions is an entire function, it follows that $u C_{\phi} f$ $=u$. $f \circ \phi \subseteq \Gamma$ for every $f \in \Gamma$. Suppose $f_{n} \rightarrow 0$ strongly. For a given $R>0$, as in proof of Theorem 2.2 choose $K>M(R, \phi)$ such that $\phi\left(\bar{D}_{R}\right) \subset \bar{D}_{K}$. Let $\varepsilon>0$ be given. Then there exists $n_{0}>0$ such that

$$
M\left(2 K, f_{n}\right)<\frac{\varepsilon}{2 M(R, u)} \quad \text { for every } n>n_{0} .
$$

From Lemma 2.1, we have

$$
\begin{aligned}
\left|u(z) f_{n}(\phi(z))\right| & \leqq M(R, u)\left|f_{n}(\phi(z))\right| \\
& \leqq M(R, u) \frac{M\left(2 K, f_{n}\right)}{2 K-|\phi(z)|} \times 2 K \\
& \leqq 2 M(R, u) M\left(2 K, f_{n}\right)<\varepsilon
\end{aligned}
$$

for each $|z| \leqq R$ and $n \geqq n_{0}$. Hence

$$
M\left(R,\left(u C_{\phi}\right)\left(f_{n}\right)\right)<\varepsilon \quad \text { for every } n \geqq n_{0}
$$

Thus $u C_{\phi} f_{n} \rightarrow 0$. This proves that $u C_{\phi}$ is continuous at origin. Since $u C_{\phi}$ is linear, so $u C_{\phi}$ is continuous everywhere. This completes the proof of the theorem. 
ACKNOWLEDGEMENTS: The authors wish to thank the refree for his valuable comments.

\section{REFERENCES}

[1] AhLfors, L. V., Complex Analysis, International Student Edition, McGraw Hill Kogakusha Ltd.

[2] Boyd, D. M., Composition operators on $H^{p}(A)$, Pacific J. Math. 62 (1976), 55-60.

[3] CarLson, JAmes, W., The spectra and commutants of some weighted composition operators, Trans, Amer. Math. Soc. 317 (1990), 631-654.

[4] Conway, John, B., Functions of one complex variable Springer International student Edition, New Delhi.

[5] Conway, John, B., A course in functional analysis, Springer Verlag, 1985.

[6] Cowen, C.C., Composition operator on $H^{2}$, J. Oper. Theory 9 (1983), 77-106.

[7] IwANiK, ANZELm, Pointwise induced operators on $L_{p}$ spaces Proc. Amer. Math. Soc. 58 (1976), 173-178.

[8] IYeR, V.G., On the space of integral functions-I, J. Indian Math. Soc. 12 (1948), $13-30$.

[9] IYer, V.G., On the space of integral functions-II, Quart J. Math. Oxford Ser (2) Vol. I (1950), 86-96.

[10] Iyer, V.G., On the space of integral functions-III, Proc. Amer. Math. Soc. Vol. 3 (1952), 874-883.

[11] Iyer, V.G., On the space of integral functions-IV, Proc. Amer. Math. Soc. 7 (1956), 644-649.

[12] MAYER, D. H., On composition operators on Banach spaces of holomorphic functions, J. Funct. Anal. 35 (1980), 191-206.

[13] NoRdgren, E. A., Composition operators on Hilbert spaces, Lecture Notes in $M_{a}$ thematics 693. Springer-Verlag, New York, 37-68.

[14] Scheefer, H.H., Topological vector spaces. The Mac Millan Company, New York, 1966.

[15] Schwartz, H. J., Composition operators on $H^{p}$, Thesis University of Toledo, 1969.

[16] SingH, R. K., Invertible composition operators on $L^{2}(\lambda)$, Proc. Amer. $M_{a}$ th. Soc. 56 (1976), 127-129.

[17] Singh, R. K. And Komal, B.S., Composition operators on $l_{p}$ and its adjoint, Proc. Amer. Math. Soc. 70 (1978), 21-25.

[18] Swantan, D. W., Composition operators on $H^{p}(D), \mathrm{Ph} . \mathrm{D}$. Thesis Northwestern University, 1974.

[19] Titchmarsh, E. C., The theory of functions, Oxford University Press, 1939.

DepartMENT OF MATHEMATics

UNIVERSITY OF JAMMU

JAMMU, JAMMU-180 001,

INDIA. 